\title{
Fatores de morbimortalidade na cirurgia eletiva do aneurisma da aorta abdominal infra-renal: experiência de 134 casos
}

\author{
Morbidity and mortality factors in the elective surgery of infrarenal \\ abdominal aortic aneurysm: a case study with 134 patients
}

\section{Aquiles Tadashi Ywata de Carvalho', Vanessa Prado dos Santos ${ }^{2}$, Álvaro Razuk Filho ${ }^{3}$, Walter Karakhaian ${ }^{3}$, Henrique Jorge Guedes Neto ${ }^{3}$, Valter Castelli Jr. ${ }^{3}$, Roberto Augusto Caffaro ${ }^{4}$}

\section{Resumo}

Contexto: O tratamento cirúrgico convencional do aneurisma da aorta abdominal (AAA) infra-renal pode resultar em complicações graves. A fim de otimizar os resultados na evolução do tratamento, é importante que sejam identificados os pacientes predispostos a determinadas complicações e instituídas condutas preventivas.

Objetivos: Avaliar a taxa de mortalidade operatória precoce, analisar as complicações pós-operatórias e identificar os fatores de risco relacionados com a morbimortalidade.

Método: Foram analisados 134 pacientes com AAA infra-renal submetidos a correção cirúrgica eletiva no período de fevereiro de 2001 a dezembro de 2005.

Resultados: A taxa de mortalidade foi de 5,2\%, sendo secundária principalmente a infarto agudo de miocárdio (IAM) e isquemia mesentérica. As complicações cardíacas foram as mais freqüentes, seguidas das pulmonares e renais. A presença de diabetes melito (DM), insuficiência cardíaca congestiva (ICC), insuficiência coronariana (ICO) e cintilografia miocárdica positiva para isquemia estiveram associadas às complicações cardíacas. A idade avançada, a doença pulmonar obstrutiva crônica (DPOC) e a capacidade vital forçada reduzida aumentaram os riscos de atelectasia e pneumonia. História de nefropatia, tempo de pinçamento aórtico prolongado e níveis de uréia elevados aumentaram os riscos de insuficiência respiratória aguda (IRA). A isquemia dos membros inferiores esteve associada ao tabagismo e à idade avançada, e a maior taxa de mortalidade, à presença de coronariopatia, tempos prolongados de pinçamento aórtico e de cirurgia.

Conclusão: A taxa de morbimortalidade esteve compatível com a literatura nacional e internacional, sendo secundária às complicações cardíacas, respiratórias e renais. Os fatores de risco identificados no pré e transoperatório estiveram relacionados com essas complicações.

Palavras-chave: Aneurisma da aorta abdominal, morbidade, mortalidade, procedimentos cirúrgicos eletivos, fatores de risco.

\begin{abstract}
Background: Conventional surgical treatment of infrarenal abdominal aortic aneurysm (AAA) can result in serious complications. To optimize treatment outcome it is important to identify patients at risk of having complications and implement prophylaxis.
\end{abstract}

Objectives: To analyze early surgical mortality rate and postsurgical complications, and to identify risk factors related to morbidity and mortality.

Method: A total of 134 patients with infrarenal AAA submitted to elective surgical correction from February 2001 to December 2005 were analyzed.

Results: The mortality rate $(5.2 \%)$ was secondary mainly to acute myocardial infarction and intestinal ischemia. Heart-related complications were the most frequent, followed by lung and kidney complications. Presence of diabetes mellitus, congestive heart failure, coronary artery disease, and scintigraphy suggestive of ischemia were related to cardiac complications. Advanced age, chronic obstructive lung disease and reduced forced vital capacity were related to higher risks of atelectasis and pulmonary infection. Presence of renal failure, prolonged aortic clamping and high urea rates were related to acute renal failure. Smoking and advanced age were associated with lower limb ischemia. Presence of obstructive coronary insufficiency and prolonged aortic clamping and surgery time were associated with higher mortality rate.

Conclusion: The morbidity and mortality rate was compatible with data found in the national and international literature, secondary to cardiac, pulmonary and kidney complications. Identified risk factors before and during the surgery were related to these complications.

Keywords: Abdominal aortic aneurysm, morbidity, mortality, elective surgical procedures, risk factors.

1. Mestre em Medicina, Faculdade de Ciências Médicas, Santa Casa de São Paulo, São Paulo, SP. Especialista em Cirurgia Vascular e Endovascular, Sociedade Brasileira de Angiologia e de Cirurgia Vascular (SBACV). Membro efetivo, SBACV.

2. Doutora em Medicina, Faculdade de Ciências Médicas, Santa Casa de São Paulo, São Paulo, SP.

3. Doutor. Professor adjunto, Faculdade de Ciências Médicas, Santa Casa de São Paulo, São Paulo, SP.

4. Doutor. Professor adjunto e chefe, Disciplina de Angiologia, Cirurgia Vascular e Endovascular, Faculdade de Ciências Médicas, Santa Casa de São Paulo, São Paulo, SP.

Este artigo é parte da tese apresentada ao Curso de Pós-Graduação da Faculdade de Ciências Médicas da Santa Casa de São Paulo, em 18 de maio de 2007, para a obtenção do título de Mestre em Medicina (membros da banca: Roberto Sacilotto, Walter Karakhanian e Adilson Ferraz Paschoa).

Não foram declarados conflitos de interesse associados à publicação deste artigo.

Artigo submetido em 14.03.07, aceito em 16.04.08.

J Vasc Bras. 2008;7(3):214-224.

Copyright@ 2008 by Sociedade Brasileira de Angiologia e de Cirurgia Vascular 


\section{Introdução}

$\mathrm{O}$ aneurisma da aorta abdominal (AAA) representa uma enfermidade importante na prática do cirurgião vascular, atingindo de 2 a $4 \%$ da população geral, em uma proporção de cinco homens para cada mulher ${ }^{1,2}$. Com o aumento da expectativa de vida, observa-se uma maior incidência do $\mathrm{AAA}^{3-5}$ : na população acima de 65 anos, a prevalência é de $6 \%{ }^{1,2}$, chegando a $10 \%{ }^{6}$ na população acima de 80 anos.

Estima-se que a incidência anual de ruptura do AAA seja de oito casos por 100.000 habitantes $^{7}$ e que o AAA seja responsável por $2 \%$ das mortes na população acima de 60 anos $^{5}$. A mortalidade desses pacientes pode estar relacionada com a ruptura do AAA, atingindo taxas entre 23 e $63 \%{ }^{2,8}$, enquanto a mortalidade operatória eletiva situa-se entre 3 e $5 \%{ }^{2,9}$.

O tratamento cirúrgico convencional caracteriza-se pela substituição do segmento aneurismático da aorta abdominal por prótese de material sintético. $\mathrm{O}$ acesso cirúrgico utilizado para a correção do AAA pode ser através das vias transperitoneal ou extraperitoneal ${ }^{10,11}$, dependendo das características anatômicas e clínicas de cada caso, com vantagens e desvantagens de acordo com a literatura ${ }^{12,13}$.

Atualmente, um procedimento menos invasivo, através da colocação de endopróteses, encontra-se cada vez mais presente na literatura, apresentando bons resultados para um determinado grupo de pacientes ${ }^{14-19}$.

As complicações pós-operatórias podem ser divididas em precoces e tardias. Entre as complicações precoces, algumas estão relacionadas com o procedimento cirúrgico, e há também complicações clínicas. As complicações imediatas mais comuns, observadas ainda durante o procedimento cirúrgico, compreendem sangramento persistente com consumo de fatores da coagulação (coagulação intravascular disseminada), lesões vasculares (principalmente lesões venosas), lesão das vísceras parenquimatosas ou ocas, lesão dos ureteres, embolia distal, trombose distal, entre outras. As complicações tardias caracterizam-se por pseudo-aneurismas anastomóticos, fístulas aorto-entéricas, trombose e infecção do enxerto ${ }^{14,20-22}$.
As complicações pós-operatórias precoces são, muitas vezes, as responsáveis pelas taxas de morbimortalidade do AAA eletivo, e por serem um reflexo do preparo pré-operatório e do estado geral do doente, merecem especial atenção em nosso estudo. Pacientes com indicação cirúrgica devem ser submetidos a avaliação préoperatória, com identificação dos fatores de risco, doenças associadas, medicações em uso e cirurgias prévias, visando à compensação das condições clínicas. A fim de otimizar o tratamento desses pacientes, acreditamos ser essencial identificar grupos de risco para determinadas complicações e avaliar a taxa de morbimortalidade, instituindo-se condutas preventivas específicas para as possíveis complicações.

Os objetivos do presente estudo foram avaliar a taxa de mortalidade operatória precoce, analisar as principais complicações (morbidades) pós-operatórias dos pacientes e identificar os fatores de risco relacionados com a morbidade e a mortalidade.

\section{Método}

Foram incluídos no estudo, consecutivamente, pacientes portadores de AAA infra-renal, submetidos a tratamento cirúrgico eletivo pela Disciplina de Cirurgia Vascular da Santa Casa de São Paulo, São Paulo, SP.

Os pacientes tiveram os aneurismas operados quando o seu diâmetro transverso superior atingiu $5 \mathrm{~cm}$ ou quando um crescimento $>0,5 \mathrm{~cm}$ foi observado em 6 meses. Não foram submetidos a tratamento cirúrgico eletivo por via convencional pacientes de risco cirúrgico muito elevado acompanhados ambulatorialmente devido a quadro de insuficiência cardíaca congestiva (ICC) de difícil tratamento, infarto agudo do miocárdio (IAM) há menos de 6 meses, angina pectoris, insuficiência renal crônica terminal, dispnéia em repouso, pacientes dependentes de oxigênio e outras enfermidades com expectativa de vida inferior a 2 anos.

Para os fins desta investigação, foram feitos registros de informações obtidas do grupo de pacientes com relação aos períodos pré, trans e pós-operatórios. Procedeu-se ao acompanhamento ambulatorial até 2 meses após a alta hospitalar. 
Realizamos então a correlação entre os fatores presentes no pré e no transoperatório e a ocorrência de complicações ou óbito no pós-operatório, buscando determinar os fatores que implicavam maior mortalidade e maior morbidade local e sistêmica.

Com relação às complicações intra e pós-operatórias, utilizou-se a técnica de regressão logística ${ }^{23}$, onde a variável de resposta é a probabilidade de um determinado paciente ter ou não uma determinada complicação, e as variáveis explicativas compreendem os diversos possíveis fatores de risco. $\mathrm{Na}$ análise, foram testadas variáveis de possíveis complicadores, e foram reportados apenas aqueles fatores que resultaram estatisticamente significantes. Um nível de significância de 0,05 foi utilizado para diferenciar fatores fortemente relevantes.

\section{Casuística}

No período de fevereiro de 2001 a dezembro de 2005, foram analisados os dados de 134 pacientes com diagnóstico de AAA infra-renal submetidos consecutivamente a correção cirúrgica eletiva, realizada na Disciplina de Cirurgia Vascular da Santa Casa de São Paulo. A idade média foi de 66,1 anos (54 e 85), e o sexo masculino representou $76,1 \%(n=102)$ dos pacientes.

A maioria dos pacientes $(73,1 \%)$ era assintomática no momento do diagnóstico, sendo a dor abdominal a sintomatologia mais referida, presente em $26,9 \%$ dos $\operatorname{casos}(\mathrm{n}=36)$.

As comorbidades associadas mais freqüentes foram: hipertensão arterial sistêmica (73,9\%), doença arterial oclusiva periférica $(64,2 \%)$, tabagismo $(56 \%)$, diabetes melito (39,6\%), dislipidemia (20,1\%), doença pulmonar obstrutiva crônica $(15,7 \%)$, insuficiência cardíaca congestiva $(14,9 \%)$, coronariopatia $(14,2 \%)$, obesidade $(9,7 \%)$ e nefropatia $(9 \%)$.

O diâmetro transverso médio do aneurisma foi de 6 $\mathrm{cm}(4,5$ a 10$)$, e o tempo da internação variou entre 3 e 60 dias.

Utilizamos como acesso cirúrgico a via extraperitoneal esquerda em $88 \%$ dos casos, seguida da transversa transperitoneal em 5,3\%, a extraperitoneal direita em $4,5 \%$ e a mediana em $2,2 \%$.

O enxerto aorto-aórtico foi realizado em $43,2 \%$ dos casos, seguido do enxerto aorto-biilíaco comum em
25,4\%, enxerto aorto-ilíaco comum e ilíaco externo em $15 \%$ e aorto-bifemoral em 9\%. Pelo menos uma das artérias ilíacas internas foi preservada em $97 \%$ dos pacientes.

O tempo cirúrgico variou entre 4 e 8 horas, com média de 5 horas, e o tempo de anestesia variou entre 4,5 e 8,5 horas, com média de 5,9 horas. Observou-se uma média de pinçamento aórtico de 1 hora $(0,5$ a 1,8).

\section{Resultados}

As morbidades pós-operatórias encontram-se listadas na Tabela 1 .

A complicação isolada mais freqüente foi a atelectasia, acometendo 36 pacientes (26,9\%), seguida da arritmia cardíaca em 21 pacientes $(15,7 \%)$ e da crise hipertensiva em 20 pacientes $(14,9 \%)$. Por outro lado, as complicações cardíacas foram as mas prevalentes $(43,2 \%)$.

Dois pacientes que apresentaram IAM (28,5\% dos óbitos) evoluíram para choque cardiogênico e óbito, e o terceiro teve boa resolução, recebendo alta após 28 dias de internação hospitalar. Observamos que $66 \%$ dos indivíduos que sofreram complicações com IAM evoluíram para o óbito.

A análise estatística revelou que os doentes com antecedentes de diabetes melito $(p=0,032)$, coronariopatia $(p=0,014)$, insuficiência cardíaca congestiva $(p=0,008)$ e alterações eletrocardiográficas no segmento ST, sugestivas de isquemia miocárdica ( $\mathrm{p}=0,033)$, apresentaram taxas elevadas de arritmia cardíaca no pós-operatório. Da mesma maneira, pacientes que apresentaram fração de ejeção $<40(p=0,011)$, cintilografia miocárdica positiva para isquemia $(\mathrm{p}=0,02)$ e insuficiência cardíaca congestiva $(p=0,045)$ foram associados com maiores taxas de edema agudo de pulmão no pós-operatório (Tabela 2).

As complicações respiratórias foram muito freqüentes, acometendo 57 doentes (42,5\%). Os fatores de risco para essas complicações encontram-se registrados na Tabela 3.

Nessa série, 11 pacientes $(8,2 \%)$ evoluíram com insuficiência renal aguda não-dialítica, e nove pacientes $(6,7 \%)$ com insuficiência renal aguda, necessitando diálise. A análise estatística mostrou que os indivíduos com história de nefropatia, ou seja, com níveis de creatinina 
Tabela 1 - Distribuição de acordo com a morbidade nos 134 pacientes submetidos a correção cirúrgica eletiva do aneurisma da aorta abdominal infra-renal pela Disciplina de Cirurgia Vascular da Santa Casa de São Paulo no período de 2001 a 2005

Morbidade

Insuficiência renal aguda não-dialítica

Insuficiência renal aguda dialítica

Atelectasia

Derrame pleural

Broncopneumonia $\mathrm{N}^{\circ}$ de pacientes $(\%)$
Embolia pulmonar

Infarto agudo do miocárdio

Arritmia

Edema agudo de pulmão

Crise hipertensiva

Infecção de ferida operatória

Hematoma de ferida operatória

Necrose de ferida operatória

Coagulopatia

Hemorragia digestiva alta

Isquemia dos membros inferiores

Isquemia da musculatura lombar

Isquemia mesentérica

Sangramento abdominal

Amputação

Sepse

Infecção urinária

Lesão venosa

Trombose venosa profunda
$11(8,2)$

$9(6,7)$

$36(26,9)$

$6(4,5)$

$10(7,5)$

$1(0,7)$

$3(2,2)$

$21(15,7)$

$14(10,4)$

$20(14,9)$

$5(3,7)$

$10(7,5)$

$2(1,5)$

$4(3)$

$2(1,5)$

$8(6)$

$2(1,5)$

$2(1,5)$

$2(1,5)$

$1(0,7)$

$3(2,2)$

$4(3)$

$6(4,5)$

$1(0,7)$

Fonte: Santa Casa de São Paulo.

e uréia $>1,6 \mathrm{mg} / \mathrm{dL}$ e $55 \mathrm{mg} / \mathrm{dL}$, respectivamente $(\mathrm{p}=$ 0,001 ), apresentaram maior risco de desenvolver disfunção renal, necessitando diálise no pós-operatório. Sendo assim, pacientes com níveis elevados de uréia $(>55$ $\mathrm{mg} / \mathrm{dL}$ ) no pré-operatório ( $\mathrm{p}=0,001)$, com tempo de pinçamento aórtico $>1,5$ hora $(\mathrm{p}=0,014)$ e com diurese $<40 \mathrm{~mL} /$ hora no intra-operatório $(\mathrm{p}=0,014)$ apresentaram maior risco de desenvolver disfunção renal não-dialítica no pós-operatório.
Complicações da ferida operatória presentes em 17 indivíduos (12,7\%) ocorreram principalmente em pacientes com acesso operatório envolvendo a região inguinal $(\mathrm{p}<0,05)$, fato que atingiu significância estatística. Quanto às possíveis correlações, o índice de massa corporal $\left(\right.$ IMC) $>30 \mathrm{~kg} / \mathrm{m}^{2}(\mathrm{p}=0,004)$ e o tempo de cirurgia $>5$ horas $(\mathrm{p}=0,045)$ estiveram associados à infecção da ferida cirúrgica $(3,7 \%)$. O IMC > $30 \mathrm{~kg} / \mathrm{m}^{2}$ 
Tabela 2 - Avaliação da relação dos fatores de risco e da morbidade cardíaca nos 134 pacientes submetidos a correção cirúrgica eletiva do aneurisma da aorta abdominal infra-renal pela Disciplina de Cirurgia Vascular da Santa Casa de São Paulo no período de 2001 a 2005

\begin{tabular}{lcc}
\hline Morbidade/Fatores associados & Relação & p \\
\hline Arritmia & \multicolumn{2}{c}{0,032} \\
DM & + & 0,014 \\
ICO & + & 0,033 \\
Alteração ECG & + & 0,008 \\
ICC & + & \\
Edema agudo de pulmão & + & 0,020 \\
CM positivo & + & 0,045 \\
ICC & - & 0,011 \\
Fração de ejeção $<40$ & &
\end{tabular}

$\overline{\mathrm{CM}}$ = cintilografia miocárdica; $\mathrm{DM}=$ diabetes melito; $\mathrm{ECG}=$ eletroencefalograma; $\mathrm{ICC}$ = insuficiência cardíaca congestiva; ICO = insuficiência coronariana.

Fonte: Santa Casa de São Paulo.

$(\mathrm{p}=0,001)$ foi relacionado com a presença de hematoma na ferida operatória (Tabela 4).

Observamos isquemia dos membros inferiores em oito pacientes $(6,0 \%)$. As causas foram embolia em cinco $(3,7 \%)$ e trombose em três $(2,3 \%)$. Os pacientes com embolia de membro inferior foram tratados com embolectomia, com boa resolução da isquemia. Dois doentes com trombose arterial de membro inferior apresentaram isquemia leve e distal (pé e pododáctilos), optando-se por tratamento clínico, com boa evolução. O terceiro paciente com trombose arterial apresentava isquemia grave do membro inferior, sendo realizada revascularização local através de enxerto fêmoropoplíteo. Esse paciente evoluiu com trombose do enxerto e isquemia irreversível e subseqüente perda da extremidade.

$\mathrm{Na}$ casuística em referência, idade $>70$ anos $(\mathrm{p}=$ $0,043)$ e histórico de tabagismo $(\mathrm{p}=0,045)$ estiveram relacionados com maior risco de complicações isquêmicas das extremidades inferiores, fato este que atingiu significância estatística.
A taxa de mortalidade no estudo foi de 5,2\% ( $=7)$, de acordo com a seguinte especificação: dois pacientes foram a óbito por IAM, dois pacientes por isquemia mesentérica, um paciente por isquemia dos membros inferiores, um paciente por insuficiência renal aguda e um paciente por broncopneumonia.

Quando se analisou a influência dos fatores de risco na mortalidade (Tabela 5), houve aumento estatisticamente significativo da mortalidade em pacientes com antecedentes de coronariopatia ( $\mathrm{p}=0,029)$, com tempos de pinçamento aórtico $>1,5$ hora $(p=0,014)$ e de cirurgia $>5$ horas $(p=0,013)$.

Ao se analisar o diâmetro transverso do aneurisma, a escolha da via de acesso cirúrgico, as perdas e a reposição volêmica no intra-operatório, não foi observada relação com taxa de mortalidade.

\section{Discussão}

Cinqüenta e oito pacientes $(43,2 \%)$ apresentaram intercorrências cardíacas, as quais representaram as principais complicações. Esse fato está de acordo com Anacleto et al., que demonstraram altas taxas de morbidade 
Tabela 3 - Avaliação da relação dos fatores de risco e da morbidade pulmonar nos 134 pacientes submetidos a correção cirúrgica eletiva do aneurisma da aorta abdominal infra-renal pela Disciplina de Cirurgia Vascular da Santa Casa de São Paulo no período de 2001 a 2005

\begin{tabular}{lcc}
\hline Morbidade/Fatores associados & Relação & p \\
\hline Atelectasia & + & 0,01 \\
DPOC & + & 0,019 \\
Tabagismo & + & 0,01 \\
IMC $>30$ & + & 0,01 \\
Derrame pleural & + & 0,041 \\
DPOC & + & 0,029 \\
Broncopneumonia & + & 0,015 \\
Idade $>$ anos & + & 0,02 \\
DPOC & + &
\end{tabular}

CVF = capacidade vital forçada; DPOC = doença pulmonar obstrutiva crônica; IMC = índice de massa corporal. Fonte: Santa Casa de São Paulo.

Tabela 4 - Avaliação da relação dos fatores de risco e complicações da ferida operatória nos 134 pacientes submetidos a correção cirúrgica eletiva do aneurisma da aorta abdominal infra-renal pela Disciplina de Cirurgia Vascular da Santa Casa de São Paulo no período de 2001 a 2005

\begin{tabular}{|c|c|c|}
\hline Morbidade/Fatores associados & Relação & $\mathbf{p}$ \\
\hline \multicolumn{3}{|l|}{ Infecção da FO } \\
\hline $\mathrm{IMC}>30$ & + & 0,004 \\
\hline Tempo de cirurgia $>5 \mathrm{~h}$ & + & 0,045 \\
\hline Incisão inguinal & + & 0,004 \\
\hline \multicolumn{3}{|l|}{ Hematoma da FO } \\
\hline $\mathrm{IMC}>30$ & + & 0,001 \\
\hline Incisão inguinal & + & 0,004 \\
\hline
\end{tabular}

FO = ferida operatória; IMC = índice de massa corporal.

Fonte: Santa Casa de São Paulo. 
Tabela 5 - Avaliação da relação dos fatores de risco e da mortalidade nos 134 pacientes submetidos a correção cirúrgica eletiva do aneurisma da aorta abdominal infra-renal pela Disciplina de Cirurgia Vascular da Santa Casa de São Paulo no período de 2001 a 200

\begin{tabular}{lcc}
\hline Fatores de risco & Relação & p \\
\hline Insuficiência coronariana & + & 0,029 \\
Tempo cirúrgico $>5$ horas & + & 0,013 \\
Tempo de pinçamento $>1,5$ hora & + & 0,014 \\
\hline
\end{tabular}

Fonte: Santa Casa de São Paulo.

e mortalidade por insuficiência cardíaca congestiva (ICC) e taquiarritmia, e com Johnston et al., que incluem a isquemia coronariana como principal complicação pós-operatória ${ }^{24,25}$. Dos 134 pacientes operados na Disciplina de Cirurgia Vascular da Santa Casa de São Paulo no período do estudo, três $(2,2 \%)$ evoluíram com IAM, sendo que dois evoluíram com choque cardiogênico e óbito ( $28,5 \%$ dos óbitos) e o terceiro teve boa evolução, recebendo alta após 28 dias de internação hospitalar. A análise estatística nos permitiu observar que os doentes com história pregressa de coronariopatia, ICC e alterações eletrocardiográficas no segmento ST apresentaram taxas elevadas de arritmia cardíaca, fato já reportado por Johnston et al., que citam uma maior incidência de arritmias em cardiopatas ${ }^{26}$.

Segundo Hertzer et al., a história de coronariopatia é fator de risco para o IAM ${ }^{27}$. Nesse estudo, a presença de IAM não esteve relacionada com fatores préoperatórios com significância estatística, fato que pode ser explicado pelo tamanho da amostra.

Intercorrências de origem pulmonar são muito freqüentes e contribuem para maiores taxas de complicações no pós-operatório. Johnston et al. relatam índices de disfunção pulmonar pós-operatória significativa em torno de $9 \%$, incluindo atelectasia, pneumonia e embolia pulmonar ${ }^{26}$. No presente caso, as complicações respiratórias foram muito freqüentes, acometendo $42,5 \%$ dos indivíduos. Um paciente apresentou sepse por infecção respiratória seguida de óbito, ao passo que outro paciente com tromboembolismo pulmonar teve boa evolução, recebendo alta no $8^{\circ}$ dia pós-operatório.

Thompson et al. mostraram que a pneumopatia e o tabagismo estiveram associados a uma maior taxa de complicações pulmonares ${ }^{28}$. Da mesma forma, esse estudo observou que fatores pré-operatórios como doença pulmonar obstrutiva crônica (DPOC), tabagismo e obesidade (IMC $>30 \mathrm{~kg} / \mathrm{m}^{2}$ ) estiveram associados a um maior risco de desenvolver complicações respiratórias no pós-operatório. Esse fato está em sintonia com Goldstone, que considera os tabagistas e os portadores de DPOC com $\mathrm{PO}_{2}<50$ e/ou $\mathrm{PCO}_{2}$ elevado ${ }^{29}$ doentes de alto risco operatório.

As complicações renais são causas importantes no aumento da morbidade e mortalidade no pós-operatório de aneurismas. Pacientes com antecedentes de nefropatia crônica, com níveis elevados de creatinina no préoperatório ou que necessitam pinçamento aórtico supra-renal no intra-operatório apresentam um maior risco de insuficiência renal ${ }^{30}$.

A ligadura da veia renal esquerda como tática operatória pode determinar certo grau de perda transitória da função renal e, quando necessária, deve ser realizada com preservação das veias gonadal e adrenal ${ }^{31}$. No estudo em questão, foi realizada a ligadura da veia renal esquerda em um indivíduo após a ocorrência de lesão inadvertida. Não foi observada piora da função renal pós-operatória.

No caso em estudo, 11 pacientes $(8,2 \%)$ evoluíram com insuficiência renal aguda não-dialítica, e nove pacientes $(6,7 \%)$ evoluíram com insuficiência renal aguda, necessitando diálise. A análise estatística mostrou que os indivíduos com história pregressa de nefropatia apresentaram maior risco de desenvolver disfunção renal no pós-operatório, necessitando diálise, fato já reportado por Batt et al., que relataram a presença de maiores taxas de complicações renais nos portadores de 
insuficiência renal prévia ${ }^{20}$. Da mesma forma, os pacientes com níveis elevados de uréia no pré-operatório, os com tempo de pinçamento aórtico prolongado e os com diurese reduzida no intra-operatório apresentaram maior risco de desenvolver disfunção renal não-dialítica no pós-operatório, fato já citado na literatura ${ }^{30-32}$.

As complicações referentes à ferida operatória aumentam a morbimortalidade cirúrgica, principalmente quando há envolvimento da prótese vascular. $\mathrm{Na}$ infecção da prótese vascular, pode ser necessária a remoção da mesma e a sutura do coto aórtico, com derivação axilo-bifemoral ${ }^{9}$.

Hallett et al. relatam 1,3\% de infecção de prótese vascular e de sangramento anastomótico ${ }^{33}$. Nessa série, cinco $(3,7 \%)$ pacientes apresentaram infecção superficial da ferida cirúrgica, quando se optou por tratamento conservador com antibioticoterapia endovenosa de amplo espectro, com boa evolução. O curto seguimento desses pacientes ( 2 meses), realizado por exames laboratoriais e de imagem, não revelou o envolvimento da prótese sintética nem indícios de infecção neste plano.

A análise estatística nos permitiu observar que os indivíduos com IMC $>30 \mathrm{~kg} / \mathrm{m}^{2}$, com tempo de cirurgia $>5$ horas e com incisão cirúrgica envolvendo a região inguinal apresentaram maiores taxas de hematomas e infecção da ferida operatória, fato já relatado por Szilagyi et al. ${ }^{34}$.

A presença de doença arterial obstrutiva periférica (DAOP) foi um fato marcante na nossa série $(64,2 \%)$. Johnston et al. também indicam uma elevada ocorrência de doença vascular periférica em doentes com AAA e enfatizam a possibilidade de complicações isquêmicas das extremidades inferiores, com risco de perda do membro $^{26}$. Diversos são os fatores que contribuem para a elevada taxa de DAOP e suas complicações nesse estudo; entre eles estão a crescente prevalência de hipertensão arterial sistêmica, diabetes melito, tabagismo, dislipidemia e a idade dos doentes, demonstrando se tratar de uma casuística composta por indivíduos com numerosos fatores de risco para aterosclerose $\mathrm{e}^{35-37}$.

Observamos uma ocorrência maior de isquemia dos membros inferiores nos indivíduos com idade avançada (> 70 anos) e nos tabagistas, tanto por embolia como por trombose arterial, sugerindo se tratar de uma população formada por doentes com fatores de risco para fenômenos tromboembólicos ${ }^{38,39}$. Por outro lado, a presença de DAOP pré-operatória, avaliada através da palpação dos pulsos periféricos, não esteve relacionada com um maior risco de isquemia dos membros inferiores.

Em um estudo realizado por Becker et al., as intercorrências ou óbitos no pós-operatório de AAA de causas gastrointestinais ocorrem basicamente devido à interrupção da vascularização intestinal, sendo que a isquemia mesentérica contribuiu com $10 \%$ dos óbitos ${ }^{9}$. Essa complicação pode ser evitada preservando-se uma das artérias ilíacas internas e com a revascularização do cólon esquerdo por meio do reimplante da artéria mesentérica inferior quando esta apresentar calibre preservado e ausência de refluxo no intra-operatório $26,33,40,41$.

Observamos isquemia mesentérica em dois pacientes $(1,5 \%)$, achado compatível com Anacleto et al., que mostraram uma incidência de 2,3\% dessa afecção no pós-operatório de $\mathrm{AAA}^{24}$. No entanto, Hallett et al., estudando 307 pacientes, revelaram uma incidência de $0,7 \%$ de isquemia do cólon ${ }^{33}$. Na nossa amostra, não foi realizada a preservação das artérias ilíacas internas nem o reimplante da artéria mesentérica inferior nos dois pacientes citados, situação que pode justificar a evolução desfavorável ${ }^{40,41}$.

Entre as complicações imediatas mais freqüentes, as lesões vasculares, principalmente as venosas, resultaram num aumento significativo da mortalidade nos pacientes operados de aneurismas de aorta. Johnston et al. reportaram 4,8\% de sangramento operatório, necessitando hemotransfusão; Diehl et al. observaram aumento da morbimortalidade em doentes com complicações hemorrágicas intra-operatórias ${ }^{26,30}$. Visando diminuir a incidência de lesões vasculares, realizamos o controle das artérias ilíacas com mínima dissecção, evitando a manipulação da face posterior e uma possível lesão venosa ${ }^{42}$.

Relatamos lesões venosas em seis pacientes (4,5\%), estando a veia renal esquerda envolvida em um dos casos, a veia cava inferior em dois, e a veia ilíaca comum em três casos. Nessas condições, observamos um aumento nos tempos de cirurgia, anestesia e volume 
infundido, porém sem acréscimo na taxa de complicações.

A taxa de mortalidade do presente estudo foi de $5,2 \%$, semelhante às taxas relatadas na literatura internacional $1^{2,8,20,24,28,30,43,44}$. No Brasil, Becker et al., estudando 600 pacientes operados eletivamente por AAA infra-renal, encontraram uma taxa de mortalidade precoce de $3,3 \%{ }^{9}$.

A idade acima de 80 anos tem sido considerada por Dardik et al. como um fator de maior preocupação nos pacientes operados por AAA, pois acarreta uma taxa de mortalidade em torno de 7 a $10 \%{ }^{43}$. Treiman et al., estudando aneurismectomia abdominal em 52 doentes octogenários, concluíram que os critérios para a indicação cirúrgica devem ser semelhantes em pacientes octogenários com boa capacidade funcional e em pacientes mais jovens ${ }^{45}$. Na nossa casuística, não houve diferença de mortalidade entre os pacientes octogenários e os mais jovens.

Em nossa série, as principais causas de óbito foram o IAM em dois pacientes e a isquemia mesentérica em outros dois. Em consonância com esse fato, Ruby et al. atribuem ao IAM a principal causa de morte no pósoperatório imediato e a longo prazo ${ }^{46}$, enquanto que Thompson et al., estudando 108 casos de AAA submetidos a correção cirúrgica eletiva, consideraram as complicações cardíacas e pulmonares ${ }^{28}$ como a principal causa de óbito. Por outro lado, diferentemente de nossa experiência, em que a sepse por broncopneumonia representou uma das causas de morte, Anacleto et al. mostraram que as complicações pulmonares representam uma importante causa de morbidade ${ }^{24}$.

A presença de insuficiência coronariana é citada por muitos autores como fator de risco para mortalida$\mathrm{de}^{8,27,30,47}$, e nossos achados evidenciaram que a presença de coronariopatia acarretou uma taxa significativamente mais elevada de morte por IAM (choque cardiogênico). Da mesma forma, nesta casuística foi registrada taxa de mortalidade maior para pacientes com tempos de pinçamento aórtico e de cirurgia prolongados, fatos já relatados por Johnston et al., que atribuíram essa ocorrência a uma maior sobrecarga cardíaca ${ }^{26}$.
Observamos que dois pacientes que não tiveram as artérias ilíacas internas preservadas apresentaram isquemia mesentérica seguida de óbito, situação já relatada por Cronenwett et al. e Longo et al. ${ }^{40,41}$. Essa ocorrência demonstrou que $100 \%$ dos nossos pacientes com isquemia intestinal evoluíram para óbito. Porém, não houve associação com significância estatística, fato atribuído ao tamanho da amostra.

De maneira similar ao que foi observado por Becker et al., quando era analisada a participação de outros fatores de risco na mortalidade, não houve aumento estatisticamente significante da mortalidade em pacientes com insuficiência renal, hipertensão arterial sistêmica, doença pulmonar obstrutiva crônica, tabagismo, diabetes melito e acidente vascular encefálico? . $^{9}$

\section{Conclusão}

Concluímos que a taxa de mortalidade foi compatível com os dados da literatura, sendo secundária principalmente ao IAM e à isquemia mesentérica; concluímos também que as complicações mais freqüentes foram as cardíacas, seguidas das pulmonares e renais.

Também foi possível observar as seguintes associações:

- Diabetes melito, ICC, ICO, alterações no ECG sugestivas de isquemia, fração de ejeção < 40 e cintilografia miocárdica positiva para isquemia foram associados a complicações cardíacas no pósoperatório, tais como arritmias e edema agudo de pulmão.

- Idade avançada, DPOC, tabagismo, etilismo, IMC > 30 e capacidade vital forçada reduzida aumentam os riscos de atelectasia, derrame pleural e broncopneumonia.

- História de nefropatia, diurese reduzida no intraoperatório, tempo de pinçamento aórtico prolongado e níveis de uréia elevados aumentam os riscos de IRA.

- Incisão envolvendo a região inguinal, IMC > 30 e tempo cirúrgico elevado aumentam as taxas de infecção e hematoma da ferida operatória.

- Tabagismo e idade avançada estão associados a isquemia dos membros inferiores. 
- Insuficiência coronariana e tempos prolongados de pinçamento aórtico e de cirurgia mostram-se associados a uma maior taxa de mortalidade.

\section{Referências}

1. Burihan E, Baptista-Silva JC. Aneurisma da aorta abdominal - como prevenir o evento fatal. Rev Soc Cardiol. Estado de São Paulo 1999;6:833-9.

2. Vincent WV. Combining abdominal aortic aneurysmectomy with gastrointestinal or biliary surgery. Am Surg. 1988;54:290-6.

3. Bengtsson H, Bergqvist D, Sternby NH. Increasing prevalence of abdominal aortic aneurysms: a necropsy study. Eur J Surg. 1992;158:19-23.

4. Breckwoldt WL, Mackey WC, O'Donnell TF. The economic implications of high risk abdominal aortic aneurysms. J Vasc Surg. 1991;13:798-804.

5. Law M. Screening for abdominal aortic aneurysms. Br Med Bull. 1998;54:903-13.

6. Bickerstaff LK, Hollier LH, Van Peenem HJ. Abdominal aortic aneurysms: the changing natural history. J Vasc Surg. 1984;1:6-12.

7. Basnyat PS, Biffin AH, Moseley LG, Hedges AR, Lewis MH. Mortality from ruptured abdominal aortic aneurysm in Wales. Br J Surg. 1999;86:765-70.

8. Crawford ES, Saleh SA, Babb III JW, Glaeser DH, Vaccaro PS, Silvers A. Infra-renal abdominal aortic aneurysm: factors influencing survival after operation performed over a 25-year period. Ann Surg. 1981;193:699-709.

9. Becker M, Bonamigo TP, Faccini FP. Avaliação da mortalidade cirúrgica em aneurismas infra-renais da aorta abdominal. J Vasc Bras. 2002;1:15-21.

10. Caffaro RA. Via de acesso para exposição ampla da coluna toracolombar anterior (T10-L5). Descrição técnica [tese de doutorado]. São Paulo: FCMSCSP; 1998.

11. Sieunarine MA, Lawrence-Brown MM, Goodman MA. Comparison of transperitoneal and retroperitoneal approaches for infrarenal aortic surgery: early and late results. Cardiovasc Surg. 1997;5:71-6.

12. Caetano Jr.O, Silva KS, Bellen BV. Vias de acesso transperitoneal e retroperitoneal em cirurgia de aorta: resultados comparativos a longo prazo. J Vasc Bras. 2005;4:227-31.

13. Sicard GA, Freeman MB, Vanderwoude JC. Comparison between the transabdominal and retroperitoneal approach for reconstruction of the infra-renal abdominal aorta. J Vasc Surg. 1987;5:19-27

14. Aun R, Saliture Neto FT, Lederman A, Waksman H. Tratamento endoluminal de aneurismas anastomóticos na aorta abdominal: relato de dois casos. J Vasc Bras. 2006;5:371-81.

15. Faries PL, Won J, Morrissey NJ. Endovascular treatment of failed prior abdominal aortic aneurysm repair. Ann Vasc Surg. 2003;17:43-8.
16. Greenberg R, Resch T, Nyman U, Lindh M, Brunkwall J, Brunkwall P. Endovascular treatment of descending thoracic aortic aneurysms: an early experience with intermediateterm follow up. J Vasc Surg. 2000;31:147-56.

17. Pereira AH, Sanvitto P, Souza GG, Costa LF, Grudtner MA. Exclusion of abdominal aortic aneurysms by aorto uni-iliac endografts. Int Angiology. 2000;19:70-9.

18. Pereira AH, Sanvitto P. Endoprótese na correção dos aneurismas da aorta abdominal. In: Pitta GBB, Castro AA, Burihan E, editores. Angiologia e cirurgia vascular: guia ilustrado. Maceió: UNCISAL/ECMAL/LAVA; 2003.

19. Yano OJ, Marin M, Hollier L. Patient selection for endovascular repair of aortoiliac aneurysms. Cardiovasc Surg. 2000;8:340-9.

20. Batt M, Staccini P, Pittaluga P, Ferrari E, Hassen-Khodja R, Declemy S. Late survival after abdominal aortic aneurysm repair. Eur J Vasc Endovasc Surg. 1999;17:338-42.

21. Szilagyi DE, Smith RF, Derusso FJ, Elliott JP, Sherrin FW. Contribuition of abdominal aortic aneurysmectomy to prolongation of life. Ann Surg. 1966;164:678-99.

22. Todd GF, Voorhees AB. Survival of elderly patients following elective abdominal aortic aneurysm resection. J Cardiovasc Surg. 1981;22:49.

23. Hosmer DW, Lemeshow S. Applied logistic regression. New York: Wiley-Interscience, 2000.

24. Anacleto G, Nobrega L, Goncalves A, et al. Aneurisma de aorta abdominal: análise de 200 casos. Rev Angiol Cir Vasc SBACVRJ. 2001;10(2).

25. Johnston KW. Nonruptured abdominal aortic anerysm:sixyear follow-up results from the multicenter prospective Canadian aneurysm study. J Vasc Surg. 1994;20:163-70.

26. Johnston KW. Multicenter prospective study of nonruptured abdominal aortic aneurysm. Part II. Variables predicting morbidity and mortality. J Vasc Surg. 1989 Mar;9(3):437-47.

27. Hertzer NR, Beven EG, Young JR. Coronary artery disease in peripheral vascular patients. Ann Surg. 1984;2:223-33.

28. Thompson JE, Hollier LH, Patman RD, Persson AV. Surgical management of abdominal aortic aneurysms: factors inflencing mortality and morbidity - a 20 year experience. Ann Surg. 1975;181:654-60.

29. Goldstone J. Aneurysms of the aorta and iliac arteries. In Moore W (editor). Vascular surgery. Philadelphia: WB Saunders; 1998. p. 435-56.

30. Diehl JT, Cali RF, Hertzer NR, Beven EG. Complications of abdominal aortic reconstrution. An analysis of perioperative risk factors in 557 patients. Ann Surg. 1983;197:49-56.

31. Silveira DR, dos Santos VP, Lamaita AF, et al. Ruptured abdominal aortic aneurysm: prognostic factors. J Vasc Br. 2005;4:321-6.

32. Hardman DT, Fisher CM, Patel MI, et al. Ruptured abdominal aortic aneurysms: who should be offered surgery? J Vasc Surgery. 1996 23:123-9.

33. Hallett Jr JW, Marshall DM, Petterson TM, et al. Graftrelated complications after abdominal aortic aneurysm repair: reassurance from a 36-year population-based experience. J Vasc Surg. 1997;25:277-84. 
34. Szilagyi DE, Elliot JP, Smith RF. Clinical fate of the patient with asymptomatic abdominal aortic aneurysm and unfit for surgical treatment. Arch Surg. 1972;104:600-6.

35. Collin J, Walton J. Is abdominal aortic aneurysm a familial disease? Br Med J. 1989;299:493.

36. Darling RC, Messina GR, Brewster DC, Ottinger LW. Autopsy study of unoperated abdominal aortic aneurysms. The case for early resection. Circulation. 1977;56(3 Suppl):II161-4.

37. White JV, Haas K, Phillips S, Comerota AJ. Adventitial elastolysis is a primary event in aneurysm formation. J Vasc Surg. 1993;17:371-81.

38. Hirose H, Ozsvath KJ, Xia S, Tilson MD. Molecular cloning of complementary DNA for additional member of the family of aortic aneurysm antigenic proteins. J Vasc Surg. 1997;26:313-8.

39. Ouriel K, Geary K, Green RM, Fiore W, Geary JE, DeWeese JA. Factors determining survival after ruptured aortic aneurysm: the hospital, the surgeon and the patient. J Vasc Surg. 1992;11:493-6.

40. Cronenwett JL, Krupski WC, Rutherford RB. Abdominal aortic and iliac aneurysms. In Rutherford RB (editor). Vascular surgery. Philadelphia: WB Saunders; 2000. p. 1246-80.

41. Longo WE, Lee TC, Barnett MG. Ischemic colitis complicating abdominal aortic aneurysm surgery in the US veteran. J Surg Res. 1996;60:351-4.
42. Carvalho ATY, Santos VP, Guedes-Neto HJ, Caffaro RA. Aspectos cirúrgicos dos aneurismas isolados das artérias ilíacas. J Vasc Bras. 2006;5:203-8.

43. Dardik A, Lin JW, Gordon TA, Williams GM, Perler BA. Results of elective abdominal aortic aneurysm repair in the 1990s: A population-based analysis of 2335 cases. J Vasc Surg. 1999 Dec;30:985-95.

44. Reigel MM, Hollier LH, Kazmier FJ. Late survival in abdominal aortic aneurysm patients: the role of selective myocardial revascularization on the basis of clinical symptoms. $\mathbf{J}$ Vasc Surg. 1987;5:222-7.

45. Treiman RL, Levine KA, Cohen JL, Cossman DV, Foran RF, Levin PM. Aneurysmectomy in the octogenarian. A study of morbidity and quality of survival. Am J Surg. 1982;144:194-7.

46. Ruby ST, Whittemore AD, Couch NP. Coronary artery disease in patients requiring abdominal aortic aneurysms repair. Ann Surg. 1985;201:758-62.

47. O'Hara PJ, Hertzer NR, Krajewski LP. Ten year experience with abdominal aortic aneurysm repair in octogenarians: early results and late complications. J Vasc Surg. 1995;21:830-8.

\section{Correspondência:}

Aquiles Tadashi Ywata de Carvalho

Rua Oito de Dezembro, 291/601, Graça

CEP 40150-000 - Salvador, BA

E-mail: aquiles_tadashi@yahoo.com.br

\section{O conteúdo do J Vasc Bras está disponivel em português e em inglês no site do Jornal Vascular Brasileiro em www.jvascbr.com.br}

